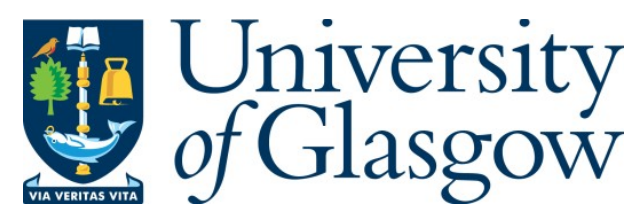

Armitage, C. J., Abdul Rahim, W., Rowe, R., and O'Connor, R. C. (2016) Trends in self-harm in Kuala Lumpur, 2005-2011. Archives of Suicide Research, 20(1), pp. 22-28.

There may be differences between this version and the published version. You are advised to consult the publisher's version if you wish to cite from it.

http://eprints.gla.ac.uk/128271/

Deposited on: 15 September 2016

Enlighten - Research publications by members of the University of Glasgow http://eprints.gla.ac.uk 
Trends in Self-Harm in Kuala Lumpur, 2005-2011

Christopher J. Armitage, PhD, University of Manchester

Wirda Abdul Rahim, PhD and Richard Rowe, PhD, University of Sheffield

Rory C. O’Connor, PhD, University of Glasgow

Address Correspondence to:

Chris Armitage

Manchester Centre for Health Psychology

School of Psychological Sciences

Manchester Academic Health Science Centre

University of Manchester

Coupland Street

Oxford Road

Manchester

M13 9PL

Tel: +44(0) 1612752556

E-mail: chris.armitage@manchester.ac.uk

Words: 1869 (excluding references)

Running head: TRENDS IN SELF-HARM IN KUALA LUMPUR, 2005-2011

Author Note. On behalf of all authors, the corresponding author states that there is no conflict of interest. We would like to thank the Ministry of Health Malaysia for funding this research. 


\section{Trends in Self-Harm in Kuala Lumpur, 2005-2011}

Acts of self-harm are not routinely tracked in Malaysia. The present study investigates the prevalence of self-harm in Kuala Lumpur, Malaysia over a 7-year period. The aims were to: (a) assess the prevalence of self-harm; (b) examine any changes over a period of seven years, and (c) identify correlates of methods of self-harm. Data were extracted from the hospital records of Kuala Lumpur Hospital to review trends in self-harm between 2005 and 2011. There were 918 episodes of self-harm across the 7-year period, with a significant peak in 2007-2009. The average rate of self-harm (7.7 per 100,000 population per year) was lower than the rate of suicide (8-13 per 100,000) suggesting that genuine cases of self-harm are often attributed to other causes. Nevertheless, over-representation of young people, women and Indians suggest areas in which resources to prevent self-harm might usefully be targeted. Estimating rates of self-harm are fraught with problems and further research is needed to understand the economic and cultural barriers around seeking treatment for self-harm, reporting self-harm and classifying self-harm.

Key words: self-harm; suicide; prevalence; Kuala Lumpur 


\section{Trends in Self-Harm in Kuala Lumpur, 2005-2011}

It is important to monitor trends in self-harm because any changes in the rates of selfharm or the demographic characteristics of the patient population could exert a substantial impact on clinical services and future suicidal behaviour $(1,2)$. However, acts of self-harm are not routinely tracked in Malaysia and just three studies have used hospital records to assess the prevalence of self-harm (3-5). The present research is timely because previous studies analysed data from the 1980's and novel because previous studies only analysed data for a period of one $(3,4)$ or two years $(5)$. For the present purposes, self-harm is defined as "any act of self-poisoning or self-injury carried out by an individual irrespective of motivation. This commonly involves self-poisoning with medication or self-injury by cutting.” (6)

The present study aims to: (a) assess the prevalence and correlates of self-harm in Kuala Lumpa, Malaysia; (b) examine any changes over a period of seven years; and (c) identify correlates of the most prevalent methods used.

\section{Method}

\section{Location}

The present research uses the records of Kuala Lumpur Hospital, from 2005 to 2011 inclusive, to estimate the prevalence of self-harm in Kuala Lumpur. Kuala Lumpur Hospital was chosen because it covers emergency admissions for the whole of Kuala Lumpur. Kuala Lumpur has a population of approximately 1.7 million, is the most densely populated area in Malaysia, and is broadly representative of Malaysia as a whole in terms of population age and ethnicity (Malaysian Statistics Department, 2010).

\section{Procedure}

The study received ethical approval from the Hospital Director, the Head of Department of the Records Office, the Ethics Committee of the National Medical Research 
Registry Malaysia, and the University’s ethics committee. Data were extracted by administrators who were independent of the research team from all records containing the International Statistical Classification of Diseases and Related Health Problems 10th Revision (7) cause codes X60-X84 for the years 2005-2011.

\section{Analyses}

Nonparametric techniques were used to analyze the data. The pattern of self-harm across years was tested using the Kolmogorov-Smirnov (K-S) statistic, which compares the rate of self-harm against a flat (uniform) distribution, where all possible outcomes are equally probable. A statistically significant $K$-S statistic indicates that the pattern deviates from uniformity. Odds ratios (OR) and 95\% confidence intervals (CI) were generated using logistic regression.

\section{Results}

\section{Comparison of People Admitted to Hospital for Self-Harm with the General Population}

Table 1 compares people admitted to hospital for self-harm with the general population.

\section{Gender}

There were significant differences in the rates of admission between men and women, $\chi^{2}(1, n=918)=69.18, p<.01$, with almost twice as many women $(n=585,63.7 \%)$ being admitted for self-harm compared with men ( $n=333,36.3 \%)$. The mean episode-based rate of self-harm per 100,000 was 2.8 per 100,000 for men and 4.2 per 100,000 for women.

\section{Age}

The distribution of self-harm across age groups differed significantly from uniformity, $K-S=16.60, p<.01$, with $777(84.6 \%)$ of the sample aged 34 years or younger (Table 2). There were significantly more 16 -24 year olds than 25-34 year olds, $\chi^{2}(1, n=675)=47.47, p$ 
$<.01$, and significantly more 25-34 year olds than 35-44 year olds, $\chi^{2}(1, n=346)=65.03, p<$ .01

\section{Ethnicity}

The distribution of self-harm across ethnic groups also differed significantly from uniformity, $K-S=8.51, p<.01$. There were significantly more Malay than Chinese, $\chi^{2}(1, n=$ $354)=56.96, p<.01$, and significantly more Indians than Malay, $\chi^{2}(1, n=682)=50.73, p<$ .01. However adjusting the figures for the proportions of ethnic groups in the population revealed significantly more self-harm cases who were classified as Indian or "other" (Table $3)$.

\section{Deaths as a Result of Self-Harm}

Twenty-four patients (2.6\%) were recorded as having died in hospital, 13 men and 11 women (1.4\% and 1.2\% of hospital admissions, respectively). More than half (54.2\%) of deaths were in the 16 to 34 age group and $62.5 \%$ of deaths were from the Indian ethnic group.

\section{Trends in Self-Harm: 2005 to 2011}

Between 2005 and 2011, 916 patients, as a result of 918 episodes, were admitted for self-harm and there appears to be a peak around 2008 (Figure 1). The distribution of selfharm across the years differed significantly from uniformity, $K-S=5.64, p<.01$. There were significant increases in self-harm between 2006 and 2007, $\chi^{2}(1, n=206)=16.33, p<.01$, and between 2007 and 2008, $\chi^{2}(1, n=304)=5.26, p=.02$, but there was a significant decrease in self-harm between 2009 and 2010, $\chi^{2}(1, n=322)=6.01, p=.01$. There were no significant differences in the rate of self-harm between the years 2005-2006, 2008-2009 or 2010-2011. Thus, admissions for self-harm peaked in the years 2007-2009.

\section{Means of Self-Harm}

Table 4 shows that the majority of self-harm was attributed to self-poisoning ( $n=$ 886), rather than other means ( $n=32)$, with “other and unspecified drugs, medicaments and 
biological substances” being the most common means $(n=486)$. Logistic regression was used to identify whether demographic correlates of of self-poisoning differed from self-harm using other means. Age, gender and ethnicity were entered as independent variables. Ethnicity emerged as the only significant predictor of poisoning, with Indians substantially more likely to engage in self-poisoning than any other ethnic group. This effect remained when all predictors were entered into the model simultaneously (Table 5).

\section{Discussion}

In the present study, we investigated trends in self-harm admissions to Kuala Lumpur Hospital. The key finding was that between 2005 and 2011 a total of 918 episodes of selfharm were recorded, with a crude rate of self-harm in Kuala Lumpur of approximately 7.7 per 100,000 population per year. Among those who self-harmed, there was: (a) an overrepresentation of younger people, women and Indians compared with the general population; (b) a peak in episodes of self-harm between 2007 and 2009 with a significant decline to 2011; and (c) a preponderance of diagnoses of intentional self-poisoning (accounting for $>90 \%$ of all self-harm episodes), which was particularly common in the Indian ethnic group.

The present rate of 131 self-harm episodes per year is lower than reported in previous studies from the same hospital, with 271 episodes in 1982 (4) and 306 episodes in 1989 (3). However, caution should be exercised when considering these figures because the self-harm rate in Kuala Lumpur is lower than the estimated suicide rate (8-13 per 100,000 population, 8). This pattern contrasts starkly with research elsewhere where self-harm is usually more common than suicide. For example, in the UK, the suicide rate in 2011 was 11.8 suicides per 100,000 population per year (9) yet the hospital-based self-harm rate rarely falls below 300 per 100,000 population per year (10).

Given that several authors have argued that the suicide rates in Malaysia have been subject to underreporting (5), it seems likely that the present self-harm rates also include 
reporting biases. Orr and Pu (4) argue that there are strong economic and cultural pressures to misclassify self-harm, from avoiding the invalidation of insurance claims to the fact that attempted suicide is a serious breach of faith for the Muslim majority. Such social and cultural pressures might also help to explain why there is an over-representation of Indians in the present data: There are conditions under which suicide is acceptable among Hindus (11).

Thus, people who have self-harmed may not seek treatment, may prefer to attend private clinics rather than those run by central government, or may claim their injury was accidental when they get to the hospital. One possible explanation for the almost 1:1 correspondence between suicide rates and self-harm rates in Malaysia is misclassification of self-harm events by health professionals. For example, in relation to completed suicides, there are persistent claims that autopsies are not routinely carried out and that deaths have been described as "severely brain injured," omitting the external cause of that condition (e.g., “intentional self-harm by jumping from a high place” [5])

It appears that, even where self-harm is diagnosed, there is a tendency to use the broader ICD-10 codes. For example, it is notable that 486/886 (54.85\%) self-poisoning episodes were attributed to X64 (“other and unspecified drugs”), 110/886 (12.4\%) selfpoisoning episodes were attributed to X69 (“other and unspecified chemicals”), and that 18/32 (56.25\%) self-harm episodes were attributed to X84 (“intentional self-harm by other means”). It would be valuable to conduct work with health professionals to understand the ways in which ICD-10 codes (particularly X60-X84) are being applied in Malaysia. It might be the case that further contextualization might be necessary to ensure that the ICD-10 is fit for purpose in the Malaysian context.

The most salient limitation of the present study relates to the quality of the data. In addition to the problems of misclassifications outlined above, a lot of data was missing from records, most notably methods implicated in self-harm, reasons for the act and treatment for 
self-harm. Future work is required to establish a more reliable body of data, and to explore the social and cultural influences on the diagnosis, classification and help-seeking following an episode of self-harm. The issue of intentionality also merits comment. Although we have adhered to the ICD terminology that describes self-poisoning and self-harm as intentional to distinguish these behaviours from accidental self-harm, our conclusions are based on the method of self-harm rather than a detailed exploration of intention. As a consequence, it is difficult to judge the extent to which the self-harm was intentional and it is also not possible to comment on how the different motives (including suicidal intent) that underpin self-harm affect recording practices $(12,13)$.

Nevertheless, there is sufficient evidence to suggest that women, younger people and members of the Indian minority may need particular help, given that they are overrepresented in the present data. Further research is required to understand the factors leading to self-harm in these sociodemographic groups. Self-poisoning among the Indian minority group might need particularly close attention from clinicians and researchers, particularly because they accounted for more than $50 \%$ of deaths due to self-harm. 


\section{References}

1. Hawton, K., Fagg, J., Simkin, S., Bale, E., \& Bond, A. (1997). Trends in deliberate selfharm in Oxford, 1985-1995. Implications for clinical services and the prevention of suicide. British Journal of Psychiatry, 171, 556-560.

2. Hawton, K., Fagg, J., Simkin, S., Bale, E., \& Bond, A. (2000). Deliberate self-harm in adolescents in Oxford, 1985-1995. Journal of Adolescence, 23, 47-55. doi: 10.1006/jado.1999.0290

3. Habil, M. H., Ganesvaran, T., \& Agnes, L. S. (1992-3). Attempted suicide in Kuala Lumpur. Asia-Pacific Journal of Public Health, 6, 5-7.

4. Orr, J. W., \& Pu, T. N. (1985). Parasuicides in Kuala Lumpur: A descriptive study. Singapore Medical Journal, 26, 161-170.

5. Ong, S., \& Yeoh, K. L. (1992). Suicidal behaviour in Kuala Lumpur. In L. P. Kok \& W. Tseng (Eds.), Suicidal behaviour in the Asia-Pacific region (pp. 144-175). Singapore: Singapore University Press.

6. National Institute for Health and Care Excellence. (2011). Self-harm: Longer-term management. Retrieved from: http://www.nice.org.uk/nicemedia/live/13619/57179/57179.pdf

7. World Health Organization (WHO). (2010). International Statistical Classification of Diseases and Related Health Problems 10th Revision (ICD-10) Version for 2010. Retrieved from: http://apps.who.int/classifications/icd10/browse/2010/en\#/X64

8. Kaur, M. (2006, October 10). Living dangerously. New Straits Times, p. 59.

9. UK Office for National Statistics (2011). Suicides in the United Kingdom, 2011. Retrieved from: http://www.ons.gov.uk/ons/dcp171778_295718.pdf 
10. Hawton, K., Casey, D., Bale, E., Rutherford, D., Bergen, H., Simkin, S., Brand, F., \& Lascelles, K. (2010). Self-harm in Oxford 2010. Retrieved from the University of Oxford, Centre for Suicide Research website: http://cebmh.warne.ox.ac.uk/csr/images/annualreport2010.pdf

11. Adityanjee, D. R. (1986). Suicide attempts and suicides in India: cross-cultural aspects. The International Journal of Social Psychiatry, 32, 64-73. doi: $10.1177 / 002076408603200208$

12. Hawton, K., Saunders, K. E. A., \& O’Connor, R. C. (2012). Self-harm and suicide in adolescents. The Lancet, 379, 2373-2382.

13. Kapur, N., Cooper, J., O’Connor, R. C., \& Hawton, K. (2013). Attempted suicide versus non-suicidal self injury: New diagnosis, or false dichotomy? British Journal of Psychiatry, 202, 326-238. 
Table 1

Comparison Between the Present Sample and the General Population

\begin{tabular}{|c|c|c|c|}
\hline Variable & $\begin{array}{l}\text { Present Sample } \\
\qquad(n=918)\end{array}$ & $\begin{array}{c}\text { Population }^{\mathrm{a}} \\
(N=1,674,621)\end{array}$ & $\begin{array}{l}\mathrm{X}^{2} \text { for difference between } \\
\text { sample and population }\end{array}$ \\
\hline Gender (\%) & & & $78.53(p<.01)$ \\
\hline Male & 36.3 & 50.9 & \\
\hline Female & 63.7 & 49.1 & \\
\hline \multicolumn{4}{|l|}{ Age (\%) } \\
\hline $0-14$ years & 2.1 & 22.1 & $213.90(p<.01)$ \\
\hline $15-64$ years & 94.3 & 73.2 & $208.98(p<.01)$ \\
\hline 65 years and older & 3.6 & 4.7 & $2.50(p=.11)$ \\
\hline \multicolumn{4}{|l|}{ Ethnicity (\%) } \\
\hline Malay & 27.0 & 45.9 & $138.63(p<.01)$ \\
\hline Chinese & 11.5 & 43.2 & $374.67(p<.01)$ \\
\hline Indian & 47.3 & 10.3 & $1355.42(p<.01)$ \\
\hline Other & 14.2 & 0.6 & $2794.73(p<.01)$ \\
\hline
\end{tabular}


Table 2

Prevalence of Self-Harm by Age

\begin{tabular}{lcc}
\hline Age (Years) & Number of Episodes & $\%$ \\
\hline 15 or younger & 32 & 3.5 \\
$16-24$ & 427 & 46.5 \\
$25-34$ & 248 & 27.0 \\
$35-44$ & 98 & 10.7 \\
$45-54$ & 48 & 5.2 \\
$55+$ & 62 & 6.8 \\
\hline
\end{tabular}

Note. 3 cases missing 
Trends in Self-harm in Kuala Lumpur, 2005-2011 12

Table 3

Prevalence of Self-Harm by Ethnicity

Ethnic Group Number of Episodes $\quad \% \quad$ Mean Annual Rate per 100,000 Population

\begin{tabular}{lccc}
\hline Malay & 248 & 27.0 & 4.61 \\
Chinese & 106 & 11.5 & 2.09 \\
Indian & 434 & 47.3 & 35.94 \\
Other & 130 & 14.2 & 184.83 \\
\hline
\end{tabular}


Table 4

Causes of Self-Harm 2005-2011

ICD-10 codes

Frequency

$\%$

X60 Intentional self-poisoning by and exposure to non-opioid

96

10.5

analgesics, antipyretics and antirheumatics

X61 Intentional self-poisoning by and exposure to antiepileptic,

64

sedative-hypnotic, anti-parkinsonism and psychotropic drugs,

not elsewhere classified

X62 Intentional self-poisoning by and exposure to narcotics and

psychodysleptics [hallucinogens], not elsewhere classified

X63 Intentional self-poisoning by and exposure to other drugs

$2<0.1$

acting on the autonomic nervous system

X64 Intentional self-poisoning by and exposure to other and

unspecified drugs, medicaments and biological substances

X65 Intentional self-poisoning by and exposure to alcohol

$5 \quad<0.1$

X66 Intentional self-poisoning by and exposure to organic solvents

$14<0.1$

and halogenated hydrocarbons and their vapours

X67 Intentional self-poisoning by and exposure to other gases and

$5<0.1$ vapours

X68 Intentional self-poisoning by and exposure to pesticides

X69 Intentional self-poisoning by and exposure to other and

unspecified chemicals and noxious substances

X70 Intentional self-harm by hanging, strangulation and

$1<0.1$ suffocation

X71 Intentional self-harm by drowning and submersion

0 0.0 
X72 Intentional self-harm by handgun discharge

$0 \quad 0.0$

X73 Intentional self-harm by rifle, shotgun and larger firearm discharge

X74 Intentional self-harm by other and unspecified firearm discharge

X75 Intentional self-harm by explosive material

X76 Intentional self-harm by smoke, fire and flames

$1<0.1$

X77 Intentional self-harm by steam, hot vapours and hot objects

X78 Intentional self-harm by sharp object

X79 Intentional self-harm by blunt object $2<0.1$

X80 Intentional self-harm by jumping from a high place $4<0.1$

X81 Intentional self-harm by jumping or lying in front of moving

$0 \quad 0.0$
object

X82 Intentional self-harm by crashing of motor vehicle

X83 Intentional self-harm by other specified means $2<0.1$

X84 Intentional self-harm by unspecified means 
Table 5

Factors Associated with Intentional Self-Poisoning $(N=918)$

\begin{tabular}{lcccc}
\hline Independent Variables & $N$ (\%) Full Sample & $n(\%)$ Not Self-Poisoning & OR (95\% CI) & Adjusted $^{\mathrm{b}}$ OR (95\% CI) \\
\hline Gender & $585(63.72)$ & $16(2.73)$ & $0.56(0.27-1.13)$ & $0.61(0.30-1.25)$ \\
Women & $333(36.28)$ & $16(4.80)$ & 1.00 & 1.00 \\
Men & & & & \\
Ethnicity & $248(27.02)$ & $12(4.84)$ & $1.29(0.51-3.24)$ & $1.25(0.50-3.16)$ \\
Malay & $106(11.55)$ & $6(5.66)$ & $1.09(0.37-3.25)$ & $1.23(0.40-3.78)$ \\
Chinese & $434(47.28)$ & $6(1.38)$ & $4.68(1.59-13.74) *$ & $4.47(1.52-13.17)^{*}$ \\
Indian & $130(14.16)$ & $8(6.15)$ & 1.00 & 1.00 \\
Other & -- & $\tau=-0.04(p=.28)$ & $0.98(0.96-1.01)$ & $0.99(0.97-1.02)$ \\
Age & &
\end{tabular}

Note. ${ }^{\mathrm{a}}$ Age (in years) was analyzed as a continuous variable. ${ }^{\mathrm{b}}$ Adjusted for gender, ethnicity, and age.

$* p<.01$ 
Figure 1

Trends in Self-Harm 2005-2011

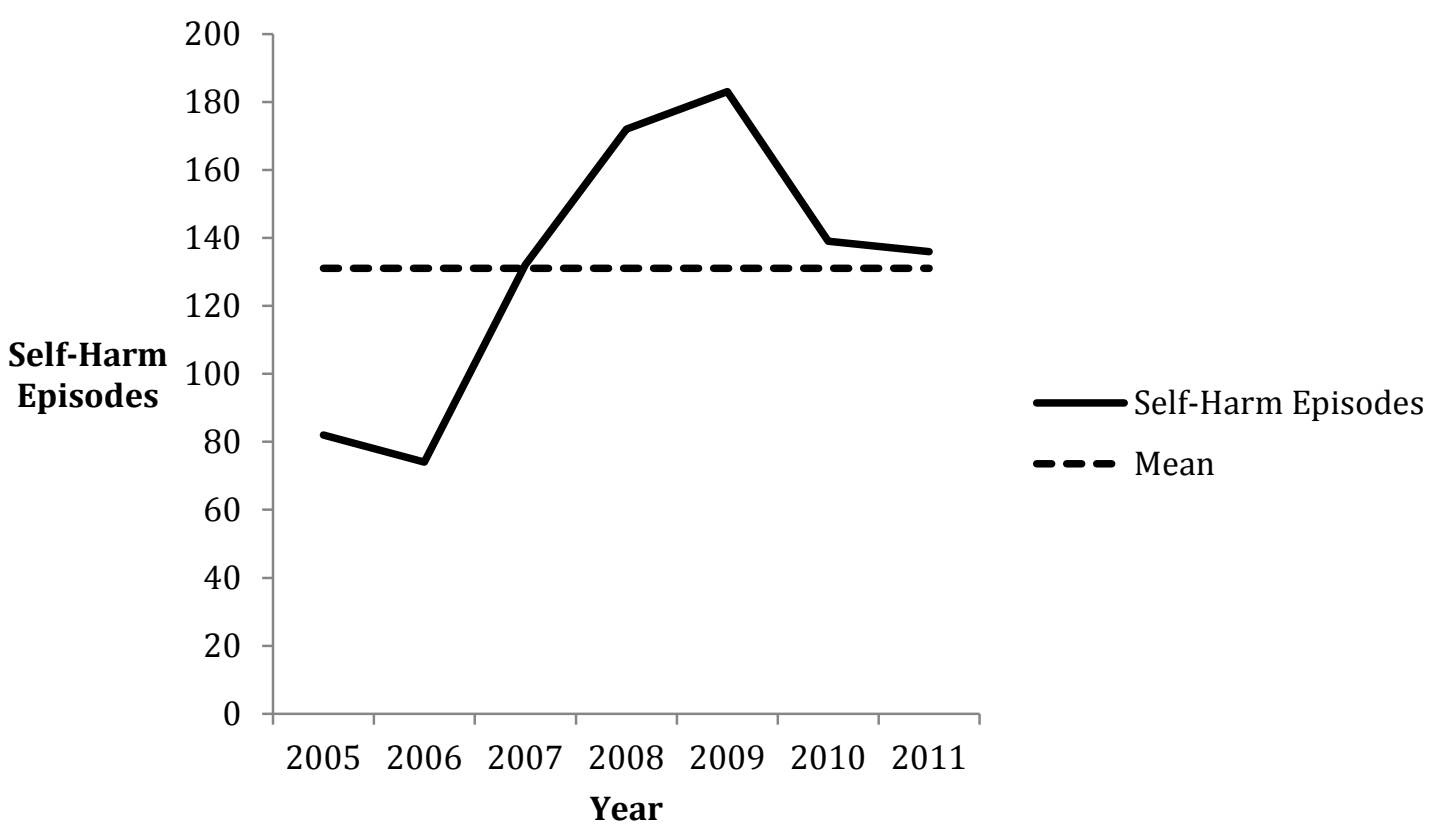

